\title{
Hyperboloidal evolution with the Einstein equations
}

\author{
Anıl Zenginoğlu \\ Max-Planck-Institut für Gravitationsphysik, Albert-Einstein-Institut, Am Mühlenberg 1, \\ D-14476 Golm, Germany
}

Received 18 June 2008, in final form 7 August 2008

Published 16 September 2008

Online at stacks.iop.org/CQG/25/195025

\begin{abstract}
We consider an approach to the hyperboloidal evolution problem based on the Einstein equations written for a rescaled metric. It is shown that a conformal scale factor can be freely prescribed a priori in terms of coordinates in a wellposed hyperboloidal initial value problem such that the location of null infinity is independent of the time coordinate. With an appropriate choice of a single gauge source function each of the formally singular conformal source terms in the equations attains a regular limit at null infinity. The suggested approach could be beneficial in numerical relativity for both wave extraction and outer boundary treatment.
\end{abstract}

PACS numbers: 04.20.Ha, 04.25.D-, 04.20.Ex, 04.30.-w

\section{Introduction}

An important problem in general relativity is the calculation of gravitational radiation emitted by self-gravitating astrophysical sources. In the isolated system idealization of such objects, one attaches to the far-field zone an asymptotic region in which the spacetime becomes flat in a certain sense. The resulting models are commonly referred to as asymptotically flat spacetimes. Because gravitational energy is not localizable and there is no generally satisfactory definition of quasi-local energy available [1], the concept of gravitational radiation is rigorously defined in these models only at null infinity [2,3]. As a consequence, one needs global access to the spacetime solution to discuss gravitational radiation in an unambiguous way. In numerical calculations, however, one typically truncates the solution domain by introducing an artificial timelike outer boundary into the spacetime. This practice introduces certain well-known conceptual and practical difficulties [4-9].

A clean way to calculate gravitational radiation is to include null infinity in the computational domain. This can be achieved under certain conditions by employing the conformal compactification technique introduced by Penrose which allows the study of null infinity by local differential geometry [10-12]. This framework has been used extensively in the mathematical discussion of isolated systems [13-17]. In numerical applications, it has been successfully applied within the characteristic approach to spacetimes which can be 
foliated by null hypersurfaces in a regular way [18-22]. In regions of highly dynamical, strong gravitational fields, however, characteristic foliations are not well behaved due to the formation of caustics in bundles of light rays generating the null hypersurfaces [23]. While there are promising suggestions on how to deal with this difficulty such as the Cauchy-characteristic matching [24-27], a successful implementation has not yet been achieved.

A more general method of including null infinity in the solution domain has been suggested by Friedrich [28]. In this approach, one solves Cauchy problems based on spacelike foliations that intersect null infinity. Such foliations are called hyperboloidal as their asymptotic behavior is similar to that of the standard hyperboloids in Minkowski spacetime. While one solves a Cauchy problem in the sense of partial differential equations, the underlying surfaces are not Cauchy surfaces in the sense of differential geometry because their domain of dependence does not cover the entire spacetime. To avoid misunderstanding, the related problem is called the hyperboloidal problem. The hyperboloidal approach is promising as the underlying surfaces combine favorable properties of standard and characteristic approaches. They are as flexible as Cauchy-type foliations commonly used in numerical relativity and they approach null infinity thus enabling a clean treatment of gravitational radiation.

In [28], the Einstein equations are reformulated for a conformally rescaled metric such that the equations are manifestly regular at null infinity. The resulting system of regular conformal field equations is larger than the Einstein equations and involves evolution equations for the conformal factor. This approach has been very efficient in the effort to understand the global structure of spacetimes [17, 29-31]. Numerical work on the hyperboloidal problem for this system has been performed mainly by Hübner [32-36], Frauendiener [37-41] and Husa $[42,43]$ (see [44] for a review). Unfortunately, the regular conformal field equations could not yet be used numerically in the study of highly dynamical systems.

In view of the recent success of numerical codes in solving the Einstein equations for a large variety of astrophysically interesting systems [45-50], it seems desirable to implement the hyperboloidal problem directly for the conformal Einstein equations [51-54]. The idea is the following: under a conformal rescaling of the metric $g=\Omega^{2} \tilde{g}$ with a function $\Omega>0$, the Ricci tensor transforms as

$$
R_{\mu \nu}[g]=R_{\mu \nu}[\tilde{g}]-\frac{1}{\Omega}\left(2 \nabla_{\mu} \nabla_{\nu} \Omega+\square \Omega g_{\mu \nu}\right)+\frac{3}{\Omega^{2}}\left(\nabla_{\lambda} \Omega\right) \nabla^{\lambda} \Omega g_{\mu \nu} .
$$

Here, $\nabla_{\mu}$ is the Levi-Civita connection of the rescaled metric $g$ and $\square:=g^{\mu \nu} \nabla_{\mu} \nabla_{\nu}$. The Einstein vacuum field equations, $G_{\mu \nu}[\tilde{g}]:=R_{\mu \nu}[\tilde{g}]-\frac{1}{2} \tilde{g}_{\mu \nu} R[\tilde{g}]=0$, are thus equivalent to a similar system for the conformally rescaled metric

$$
G_{\mu \nu}[g]=T_{\mu \nu}[\Omega]:=-\frac{2}{\Omega}\left(\nabla_{\mu} \nabla_{\nu} \Omega-\square \Omega g_{\mu \nu}\right)-\frac{3}{\Omega^{2}}\left(\nabla_{\lambda} \Omega\right) \nabla^{\lambda} \Omega g_{\mu \nu} .
$$

To include null infinity in the computational domain, one allows the conformal factor $\Omega$ to vanish in a suitable way and solves the above system directly. It has been pointed out [55] that there are two major difficulties in this program, even if one assumes that the conformal extension is regular. First there is the question of how to fix $\Omega$. The conformal factor is related to the asymptotic structure of the spacetime, therefore it must be determined jointly with the metric. Secondly, there are terms involving divisions by powers of $\Omega$ which are formally singular at $\{\Omega=0\}$. The question then is how to make sure that these terms attain regular limits at null infinity.

Assuming a regular conformal extension, these difficulties have been solved in the characteristic case by Tamburino and Winicour [56]. Their solution includes a certain choice of coordinate and conformal gauge in which the formally singular terms in (2) attain regular limits at null infinity. Their construction provides, in a sense, the mathematical basis for 
characteristic codes that include null infinity in the computational domain. The problem in the hyperboloidal case has been open.

We address the above-mentioned difficulties in two propositions. The first one treats the case of a strictly positive conformal factor and states that it can be prescribed almost arbitrarily in terms of local spacetime coordinates in a well-posed Cauchy problem for the Einstein equations. For the second proposition we allow the conformal factor to vanish in a certain way so that null infinity can be included in the computational domain. We see that a class of gauges can be chosen in a hyperboloidal initial value problem in such a way that the formally singular terms due to conformal compactification attain regular limits at null infinity for a certain class of asymptotically flat spacetimes.

\section{A hyperbolic reduction for a positive conformal factor}

The system (2) has the form of Einstein equations with source terms. In general, such a system must be completed by additional equations derived from the Bianchi identities, $\nabla^{\mu} G_{\mu \nu}=\nabla^{\mu} T_{\mu \nu}=0$, implying equations of motion for the source functions. In our case, however, there are no additional equations required for the conformal factor $\Omega$ as shown below.

Proposition 1. The conformal Einstein equations (2) admit a well-posed initial value problem for an arbitrary $\Omega \in C^{3}\left(\mathbb{R}^{4}, \mathbb{R}_{+}\right)$.

First we show that the Bianchi identities are satisfied for a positive, sufficiently differentiable conformal factor. We calculate

$$
\begin{aligned}
\nabla^{\mu} T_{\mu \nu}[\Omega]= & -\frac{2}{\Omega^{2}} \nabla^{\mu} \Omega\left(2 \nabla_{\mu} \nabla_{\nu} \Omega+\square \Omega g_{\mu \nu}\right) \\
& -\frac{2}{\Omega}\left(\square \nabla_{\nu} \Omega-\nabla_{\nu} \square \Omega\right)+\frac{6}{\Omega^{3}}\left(\nabla_{\nu} \Omega\right)\left(\nabla_{\lambda} \Omega\right) \nabla^{\lambda} \Omega .
\end{aligned}
$$

Contracting the commutation relation $\nabla_{\lambda} \nabla_{\nu} \nabla_{\rho} \Omega-\nabla_{\nu} \nabla_{\lambda} \nabla_{\rho} \Omega=R_{\lambda \nu \rho}{ }^{\sigma} \nabla_{\sigma} \Omega$ with $g^{\lambda \rho}$ and exchanging derivatives we get $\square \nabla_{\nu} \Omega-\nabla_{\nu} \square \Omega=R_{v}^{\sigma} \nabla_{\sigma} \Omega$. Using this relation, equation (2) with the definition of the Einstein tensor $G_{\mu \nu}[g]$ and the conformal source tensor $T_{\mu \nu}[\Omega]$, we get

$$
\nabla^{\mu} T_{\mu \nu}[\Omega]=-\frac{2}{\Omega} \nabla^{\mu} \Omega\left(G_{\mu \nu}[g]-T_{\mu \nu}[\Omega]\right)=0 .
$$

We see that the Bianchi identities are satisfied by virtue of the conformal Einstein equations for a non-vanishing $\Omega$ that is at least three times differentiable, i.e. $\Omega \in C^{3}\left(\mathbb{R}^{4}, \mathbb{R}_{+}\right)$, but is otherwise arbitrary. Therefore, $\Omega$ can be regarded as a free function. We can write down some suitable equation for it consistent with the above calculation, or prescribe it directly in terms of some yet unspecified coordinate system as long as $\Omega \neq 0$.

The next step is to set up a well-posed initial value problem for (2). We employ the general wave gauge reduction of the Einstein equations, also known as the generalized harmonic reduction for historical reasons, where the argument of well-posedness for (2) can be taken almost directly from $[57,58]$ with only a minor modification.

Regarding the Ricci tensor $R^{\mu \nu}$ as a differential operator acting on the metric $g$, we can write the conformal Einstein equations in a local coordinate system $\left\{x^{\mu}\right\}_{\mu=0,1,2,3}$ as

$R^{\mu \nu}[g]=\frac{1}{2} g^{\lambda \rho} \partial_{\lambda} \partial_{\rho} g^{\mu \nu}+\nabla^{(\mu} \Gamma^{\nu)}-g^{\lambda \rho} g^{\sigma \tau} \Gamma_{\lambda \sigma}^{\mu} \Gamma_{\rho \tau}^{\nu}=T^{\mu \nu}-\frac{1}{2} g_{\mu \nu} T$

where we have defined the contracted Christoffel symbols $\Gamma^{\mu}:=g^{\sigma \tau} \Gamma_{\sigma \tau}^{\mu}=-\square x^{\mu}$, set $\nabla^{\mu} \Gamma^{\nu}=g^{\mu \rho}\left(\partial_{\rho} \Gamma^{\nu}+\Gamma_{\rho \lambda}^{\nu} \Gamma^{\lambda}\right)$ and $T=g^{\lambda \rho} T_{\lambda \rho}$. The principal part of the operator $R^{\mu \nu}$ is of no known type. It was recognized by Choquet-Bruhat [59] that one can always choose a wave 
gauge, historically referred to as a harmonic gauge, at least locally, so that the contracted Christoffel symbols vanish, $\Gamma^{\mu}=-\square_{g} x^{\mu}=0$, and the system (5), reduces to a quasi-linear system of wave equations. This reduction technique led to the first local existence result in general relativity [59].

The reduction based on the wave gauge was generalized to arbitrary coordinate systems by Friedrich with the introduction of gauge source functions [60]. In the general wave gauge, the coordinates are constructed as solutions to an initial value problem for the semi-linear system of wave equations $\square_{g} x^{\mu}=-\Gamma^{\mu}=-F^{\mu}$ with prescribed functions $F^{\mu}(x, g)$ that can depend on the coordinates and the metric. These functions act as source functions for the coordinate gauge, hence the name gauge source functions. Note that the general wave gauge, in contrast to the wave gauge described above, is not a specific choice of coordinates but a particular way to prescribe general coordinates in an initial value problem.

The reduced system for (5) is then obtained by replacing the contracted Christoffel symbols with the gauge source functions $F^{\mu}$. The result is a quasi-linear system of wave equations for the metric components which can be written as

$$
G^{\mu \nu}[g]=T^{\mu \nu}[\Omega]+\nabla^{(\mu} C^{\nu)}-\frac{1}{2}\left(\nabla_{\lambda} C^{\lambda}\right) g^{\mu \nu},
$$

where $C^{\mu}=\Gamma^{\mu}-F^{\mu}$ are called the constraint fields. We want to study the Cauchy problem for this system. We will only point out certain aspects that play a role in later considerations or that are different from the detailed discussion in [58].

The Cauchy data on an initial hypersurface $\mathcal{S} \equiv\left\{x^{0}=0\right\}$ consist of $\left.g^{\mu v}\right|_{\mathcal{S}}$ and $\left.\partial_{0} g^{\mu \nu}\right|_{\mathcal{S}}$. Assume we are given on $\mathcal{S}$ a Riemannian metric $h_{a b}$ and a symmetric tensor field $K_{a b}$ as a solution to the Einstein constraint equations where $a, b=1,2,3$. We choose gauge source functions $F^{\mu}\left(x^{\lambda}\right)$ and four functions on $\mathcal{S}$ that correspond to initial data for the lapse function $\alpha>0$ and the three components of the shift vector $\beta^{a}$. In the interior, these functions should be chosen such that $\partial_{0}$ is timelike which implies $\alpha^{2}-h_{a b} \beta^{a} \beta^{b}>0$. We will later allow $\partial_{0}$ to become null at the outer boundary (see the discussion leading to (12)). We obtain the data $\left.g^{\mu \nu}\right|_{\mathcal{S}}$ via the decomposition

$$
g=g^{\mu \nu} \partial_{\mu} \partial_{\nu}=-\frac{1}{\alpha^{2}} \partial_{0}^{2}+\frac{2}{\alpha^{2}} \beta^{a} \partial_{0} \partial_{a}+\left(h^{a b}-\frac{\beta^{a} \beta^{b}}{\alpha^{2}}\right) \partial_{a} \partial_{b} .
$$

The data $\left.\partial_{0} g^{\mu v}\right|_{\mathcal{S}}$ are determined such that $\left.C^{\mu}\right|_{\mathcal{S}}=0$ and $K_{\alpha \beta}$ is the second fundamental form on $\mathcal{S}$. Standard theorems guarantee that we can find a unique solution to the Cauchy problem for the reduced equations (6) that depends continuously on the initial data. The solution spaces of (6) and (2) are equivalent if the constraint fields vanish. The Bianchi identity, $\nabla_{\mu} G^{\mu \nu}=0$, and (4) together with (6) imply the following subsidiary system for the constraint fields:

$$
\square C^{\mu}+R_{\nu}^{\mu} C^{\nu}-\frac{4}{\Omega} \nabla_{\nu} \Omega\left(\nabla^{(\mu} C^{\nu)}-\frac{1}{2}\left(\nabla_{\lambda} C^{\lambda}\right) g^{\mu \nu}\right)=0 .
$$

Initial data for the evolution equations have been constructed such that $\left.C^{\mu}\right|_{\mathcal{S}}=0$. From the evolution equations evaluated on $\mathcal{S}$ it follows that $\left.\partial_{0} C^{\mu}\right|_{\mathcal{S}}=0$. The uniqueness of solutions to the Cauchy problem for the semi-linear, homogeneous system of wave equations for $C^{\mu}$ given in (8) then implies that the solution to the reduced system (6) satisfies $C^{\mu}=0$ away from the initial surface $\mathcal{S}$. Thus we have shown that there is a well-posed hyperbolic reduction for the conformal Einstein equations (2).

We shall briefly elaborate on how the free prescription of the conformal factor is to be understood. We cannot prescribe the conformal factor as a function on the manifold because, in an initial value problem, we do not know the manifold. The prescription of a function for the conformal factor determines only its representation in terms of coordinates which are yet to be constructed during the solution process. Invariant properties of the resulting conformal 
factor will depend on initial data and the choice of gauge source functions. The essential property of (2) that is responsible for this feature is its conformal invariance, in the sense that if $(\widetilde{\mathcal{M}}, g, \Omega)$ is a solution to $G_{\mu \nu}[g]=T_{\mu \nu}[\Omega]$, then $\left(\widetilde{\mathcal{M}}, \omega^{2} g, \omega \Omega\right)$ with a positive function $\omega$ is a solution to $G_{\mu \nu}\left[\omega^{2} g\right]=T_{\mu \nu}[\omega \Omega]$. The system (2) determines the conformal class of the metric $g$ in contrast to the Einstein equations which determine the isometry class of $\tilde{g}$. This allows us to prescribe an arbitrary coordinate representation for the conformal factor as long as there are no geometric requirements to be satisfied.

\section{Choice of gauge at null infinity}

In this section, we deal with the formally singular terms in the conformal source tensor $T_{\mu \nu}[\Omega]$. We first present a preferred conformal gauge at null infinity in which each of the conformal source terms attains a regular limit at null infinity in a given conformal extension. Then we present how this choice of gauge can be achieved by a suitable choice of gauge source functions in a hyperboloidal initial value problem where the conformal factor has been prescribed explicitly in terms of coordinates. We restrict our attention to future null infinity, denoted by $\mathscr{I}^{+}$. The treatment of past null infinity follows by time reversal.

\subsection{The preferred conformal gauge at $\mathscr{I}^{+}$}

Assume that a solution $(\widetilde{\mathcal{M}}, \tilde{g})$ to the Einstein vacuum field equations has been given which admits a conformal extension $(\mathcal{M}, g, \Omega)$ including a smooth piece of $\mathscr{I}^{+}$. The existence of a broad class of such solutions is due to [28,61]. It has also been shown that solutions exist which not only admit a smooth piece of $\mathscr{I}^{+}$but a complete $\mathscr{I}^{+}$[62] (see also [29, 63, 64]). We show that $\mathscr{I}^{+} \subset\{\Omega=0, \mathrm{~d} \Omega \neq 0\}$ is a shear-free null surface independent of the conformal gauge [12, 65]. Multiplying (2) with $\Omega^{2}$ and evaluating it along $\mathscr{I}^{+}$we see that $\left.g^{\lambda \rho} \nabla_{\lambda} \Omega \nabla_{\rho} \Omega\right|_{\mathscr{I}^{+}}=0$. This shows together with $\left.\mathrm{d} \Omega\right|_{\mathscr{I}^{+}} \neq 0$ that $\mathscr{I}^{+}$is a null surface. Now multiply (2) with $\Omega$ and take its trace-free part along $\mathscr{I}^{+}$to get

$$
\left.\left(\nabla_{\mu} \nabla_{\nu} \Omega-\frac{1}{4} g_{\mu \nu} \square \Omega\right)\right|_{\mathscr{I}^{+}}=0
$$

The relation above is independent of the conformal gauge because we derived it from the conformal transformation behavior of the Einstein tensor (2). Another way to see the conformal invariance of (9) is to consider the transformation behavior of (9) under a further rescaling of the conformal metric given by

$$
g^{\prime}=\omega^{2} g, \quad \Omega^{\prime}=\omega \Omega, \quad \omega>0 \text { on } \mathcal{M} .
$$

We have $\nabla_{\mu}^{\prime} \nabla_{\nu}^{\prime} \Omega^{\prime}{\mid \mathscr{I}^{+}}=\omega \nabla_{\mu} \nabla_{\nu} \Omega+g_{\mu \nu} \nabla^{\lambda} \Omega \nabla_{\lambda} \omega$. The trace of this relation reads

$$
\square^{\prime} \Omega^{\prime}{\mid \mathscr{I}^{+}}=\frac{1}{\omega^{2}}\left(\omega \square \Omega+4 \nabla^{\lambda} \Omega \nabla_{\lambda} \omega\right),
$$

whence we get

$$
\left.\left(\nabla_{\mu}^{\prime} \nabla_{\nu}^{\prime} \Omega^{\prime}-\frac{1}{4} g_{\mu \nu}^{\prime} \square^{\prime} \Omega^{\prime}\right)\right|_{\mathscr{I}^{+}}=\left.\omega\left(\nabla_{\mu} \nabla_{\nu} \Omega-\frac{1}{4} g_{\mu \nu} \square \Omega\right)\right|_{\mathscr{I}^{+}}=0 .
$$

To see that (9) implies shear-freeness of $\mathscr{I}^{+}$, we introduce in a neighborhood of $\mathscr{I}^{+}$a null vector field $l^{\mu}$ that satisfies $\left.l^{\mu}\right|_{\mathscr{I}^{+}}=\nabla^{\mu} \Omega$. We complete $l^{\mu}$ to a Newman-Penrose complex null tetrad $(l, k, m, \bar{m})$ satisfying the usual relations [66]. Newman and Penrose introduced 12 complex functions called spin coefficients. We are interested in two of them, namely $\sigma:=m^{\mu} m^{\nu} \nabla_{\mu} l_{v}$ and $\rho:=m^{\mu} \bar{m}^{\nu} \nabla_{\mu} l_{\nu}$. As discussed in [66], when $l^{\mu}$ is tangent to an affinely parametrized null geodesic, $\sigma$ can be interpreted as the complex shear of the null geodesic 
congruence given by $l^{\mu}$ and the expansion of the congruence is characterized by $\rho$. We see with (9)

$$
\sigma{\mid \mathscr{I}^{+}}=\frac{1}{4} m^{\mu} m^{v} g_{\mu \nu} \square \Omega=0,\left.\quad \rho\right|_{\mathscr{I}^{+}}=\frac{1}{4} m^{\mu} \bar{m}^{v} g_{\mu \nu} \square \Omega=\frac{1}{4} \square \Omega .
$$

In our case, the null generators of $\mathscr{I}^{+}$are not necessarily geodesic, that is, in general they do not satisfy $l^{\lambda} \nabla_{\lambda} l^{\mu}=0$ on $\mathscr{I}^{+}$. However, under a rescaling of $l^{\mu}$ given by $\left(l^{\prime}\right)^{\mu}=\theta l^{\mu}$ with a positive function $\theta$, the spin coefficient $\sigma$ transforms as $\sigma^{\prime}=\theta \sigma$, so the vanishing of $\sigma$ is invariant under such a rescaling which we can use to make $l^{\mu}$ geodesic. We conclude that $\mathscr{I}^{+}$ is a shear-free surface in any conformal gauge.

While the vanishing of $\sigma$ and thus the shear-freeness of $\mathscr{I}^{+}$is conformally invariant, the vanishing of the expansion of $\mathscr{I}^{+}$, characterized by $\left.\square \Omega\right|_{\mathscr{I}^{+}}$, depends on the conformal gauge. As can be seen from (11), given a conformal extension we can always find a rescaling (10) such that $\square^{\prime} \Omega^{\prime}{\mid \mathscr{I}^{+}}=0$ by solving the ordinary differential equation $\nabla^{\lambda} \Omega \nabla_{\lambda} \ln \omega{\mid \mathscr{I}^{+}}=-\left.\frac{1}{4} \square \Omega\right|_{\mathscr{I}^{+}}$.

We call the conformal gauge in which the expansion of $\mathscr{I}^{+}$vanishes a preferred conformal gauge. This gauge has been useful in mathematical studies because of its special properties $[65,67]$. It is also the gauge choice of Tamburino and Winicour [56]. By (9) and (2) we see that in a preferred conformal gauge

$$
\left.\nabla_{\mu} \nabla_{\nu} \Omega\right|_{\mathscr{I}^{+}}=0, \quad \text { and } \quad \lim _{\Omega \rightarrow 0} \frac{1}{\Omega} g^{\lambda \beta} \nabla_{\lambda} \Omega \nabla_{\beta} \Omega=0
$$

which implies that each conformal source term in (2) attains a regular limit at $\mathscr{I}^{+}$.

\subsection{The choice of gauge source functions at $\mathscr{I}^{+}$}

The above construction of a preferred conformal gauge assumes that a conformal extension have been given. We are interested, however, in the case where only initial data have been given and we would like to know how to choose the gauge source functions suitably so that the hyperboloidal evolution is performed in a preferred conformal gauge.

Proposition 2. Assume hyperboloidal initial data have been given whose evolution admits a smooth conformal compactification at $\mathscr{I}^{+}$. A preferred conformal gauge can be achieved in a general wave gauge reduction of (2) by using $\Omega$ as a coordinate near $\{\Omega=0\}$ and choosing the related gauge source function $F^{\Omega}$ such that $\left.F^{\Omega}\right|_{\{\Omega=0\}}=0$.

We can use the conformal factor as a coordinate near $\{\Omega=0\}$ because $\left.\mathrm{d} \Omega\right|_{\{\Omega=0\}} \neq 0$. In the general wave gauge, there is a gauge source function related to each coordinate via $\square_{g} x^{\mu}=-F^{\mu}$. Using the conformal factor as a coordinate, we see that the value of the gauge source function $F^{\Omega}=-\square \Omega$ at $\mathscr{I}^{+}$is a direct measure of the expansion of $\mathscr{I}^{+}$. Setting it to zero makes the expansion vanish and hence gives a preferred conformal gauge.

While its proof is very simple, proposition 2 is a remarkable property of the conformal Einstein equations. In general, it is not known how a desirable gauge can be achieved by a suitable choice of gauge source functions [68] because geometric properties of coordinates depend not only on this choice but also on initial data in an essentially nonlinear way. The above proposition is very special in this respect as it states that one can, by a suitable choice of a single gauge source function, fix an a priori known coordinate surface to be a null surface free of shear and expansion that corresponds to null infinity.

In a practical numerical calculation one may proceed as follows. One calculates on a three-dimensional surface $\mathcal{S}$ with a two-dimensional boundary $\Sigma$ hyperboloidal initial data $\left(\mathcal{S}, h_{a b}, K_{a b}\right)$ whose evolution admits a smooth conformal compactification at $\mathscr{I}^{+}$. The existence of such data has been studied in [61]. In the case of isotropic extrinsic curvature, that is, under the assumption $K_{a b}=\frac{K}{3} h_{a b}$, the data need to be such that the trace-free part 
of the second fundamental form induced by $h_{a b}$ on $\Sigma$ vanishes. This condition corresponds to shear-freeness of $\mathscr{I}^{+}$. The case with more general data has been considered in [69, 70]. One can construct the data in such a way that the conformal factor has the form $\Omega=1-r$ in a neighborhood of $\Sigma$ where $r$ is a coordinate whose level surfaces have spherical topology [52]. The form of the conformal factor in the interior can be chosen freely to satisfy practical needs, for example to obtain high resolution in certain domains. Initial data for lapse and shift should be chosen such that $\Omega^{\mu}{\mid \mathscr{I}^{+}}=-\left.g^{\mu r}\right|_{r=1}=\partial_{0}^{\mu}=\delta_{0}^{\mu}$, which implies with (7)

$$
\left.\alpha\right|_{\Sigma}=\sqrt{h^{r r}},\left.\quad \beta^{a}\right|_{\Sigma}=-h^{a r} .
$$

The gauge source functions can be given quite freely as long as the condition $\left.F^{r}\right|_{r=1}=0$ is satisfied. Then each of the conformal source terms in

$$
T_{\mu \nu}=-\frac{2}{1-r}\left(\Gamma_{\mu \nu}^{r}-g_{\mu \nu} F^{r}\right)-\frac{3}{(1-r)^{2}} g_{\mu \nu} g^{r r},
$$

will attain a regular limit at $\mathscr{I}^{+}$and the system (2) can, in principle, be solved. In practice, however, the accurate calculation of these limits poses a major computational difficulty that needs further study.

\section{Discussion}

We studied the hyperboloidal evolution problem for the conformal Einstein equations. Two propositions have been made that suggest an alternative method for numerical calculations of asymptotically simple spacetimes including null infinity, substantiating earlier expectations in this direction [51-54].

Proposition 1 states that one can formulate a well-posed initial value problem for the Einstein equations in which a positive conformal factor is prescribed freely in terms of coordinates. In a sense, this result can be regarded as trivial because a rescaling with a positive factor essentially amounts to a relabeling of the metric. On the other hand, the possibility of prescribing the conformal factor in a well-posed initial value problem is very convenient because it allows us to set up a formulation of the Einstein equations for a rescaled metric in which the conformal factor is independent of the time coordinate in the whole solution domain. Depending on practical demands, one can choose the conformal factor in certain regions to be unity so that the standard Einstein equations are obtained, or larger than unity for refinement.

Proposition 2 shows how one can construct the solution to a hyperboloidal initial value problem directly in a preferred conformal gauge by a suitable choice of a single gauge source function. In this gauge the conformal source terms attain regular limits at null infinity. In combination with proposition 1 , this allows us to include null infinity in the solution domain in such a way that the outer boundary coincides with null infinity. It is a remarkable feature of the conformal Einstein equations in the general wave gauge that the constraint equations, the evolution system, the gauge system and the subsidiary system all work in harmony with conformal transformation formulae allowing us to make the geometric properties of null infinity manifest on an a priori known coordinate surface.

We have assumed vacuum throughout the calculations. Matter can be included by solving the matter equations with respect to the conformally rescaled metric if the matter fields remain confined to a finite region of space or fall off sufficiently fast towards infinity.

The approach presented in this paper has various advantages that are favorable for numerical calculations. First of all, the method is tailored for the treatment of the asymptotic region such that the interior scheme is not necessarily modified. In fact, hyperboloidal 
foliations can be made to coincide with Cauchy-type foliations in the interior where the conformal factor can be set to unity if desired [71]. In addition, the principal part of the equations to be solved in the exterior domain is identical to the standard Einstein equations which we know how to treat numerically for a large class of astrophysically interesting configurations. Furthermore, the location of null infinity is known a priori simplifying numerical outer boundary treatment and wave extraction significantly. The calculation of the news function is in our coordinates very simple, because the solution is obtained directly in Bondi coordinates at null infinity in contrast to the characteristic approach where the freedom in the null coordinates is fixed in the interior of the spacetime [72, 73].

A successful implementation of the hyperboloidal approach would solve two major problems in numerical relativity, namely the outer boundary problem and the wave extraction problem. One should be aware, however, that many promising ideas appeared during the history of numerical relativity which encountered major difficulties in practical implementation that remain unsolved. In our case, the most delicate issue is whether the formally singular terms can be numerically calculated in a stable manner. This question is open and is left for future work. We emphasize, however, that the viability of a similar calculation was demonstrated within the characteristic approach a long time ago [74].

It should be pointed out that there is a theoretical limitation to the hyperboloidal approach. It does not allow us to calculate global spacetimes because spatial infinity is not included in the computational domain. A detailed study of the asymptotic behavior of gravitational fields near spatial infinity is difficult and an active area of research [64, 75-78]. It seems that one should first achieve a successful implementation of the hyperboloidal problem for astrophysically interesting configurations before attacking the more challenging problem of spatial infinity.

\section{Acknowledgments}

I thank Sascha Husa for suggesting the research project. I am grateful to Helmut Friedrich and Sascha Husa for guidance into the conformal approach and comments on the manuscript. I would also like to thank Robert Beig, Carsten Schneemann and Jeffrey Winicour for discussions.

\section{References}

[1] Szabados L B 2004 Living Rev. Rel. 74

[2] Bondi H, van der Burg M G J and Metzner A W K 1962 Proc. R. Soc. A 269 21-52

[3] Sachs R K 1962 Proc. R. Soc. A 270 103-26

[4] Pazos E, Dorband E N, Nagar A, Palenzuela C, Schnetter E and Tiglio M 2007 Class. Quantum Grav. 24 S341-68 (Preprint gr-qc/0612149)

[5] Rinne O, Lindblom L and Scheel M A 2007 Class. Quantum Grav. 24 4053-78 (Preprint arXiv:0704.0782)

[6] Buchman L T and Sarbach O C A 2007 Class. Quantum Grav. 24 S307-26 (Preprint gr-qc/0703129)

[7] Sarbach O and Tiglio M 2005 J. Hyp. Diff. Equ. 2 839-83 (Preprint gr-qc/0412115)

[8] Friedrich H and Nagy G 1999 Commun. Math. Phys. 201 619-55

[9] Friedrich H 2005 Ann. Phys. 15 84-108 (Preprint gr-qc/0508016)

[10] Penrose R 1963 Phys. Rev. Lett. 10 66-8

[11] Penrose R 1964 Relativity, Groups and Topology ed C DeWitt and B DeWitt (New York: Gordon and Breach) pp 565-84

[12] Penrose R 1965 Proc. R. Soc. A 284 159-203

[13] Hawking S W and Ellis G F R 1973 The Large Scale Structure of Spacetime (Cambridge: Cambridge University Press)

[14] Geroch R 1977 Asymptotic Structure of Space-Time ed F Esposito and L Witten (New York: Plenum) pp 1-105

[15] Ashtekar A 1980 General Relativity and Gravitation: One Hundred Years After the Birth of Albert Einstein ed A Held (New York: Plenum) vol 2 pp 1-36 
[16] Beig R and Schmidt B 1982 Commun. Math. Phys. 87 65-80

[17] Friedrich H 1992 Recent Advances in General Relativity ed A Janis and J Porter (Basel: Birkhauser) pp 146-81

[18] Gómez R, Papadopoulos P and Winicour J 1994 J. Math. Phys. 354184 (Preprint gr-qc/0006081)

[19] Bartnik R A and Norton A H 1999 Preprint gr-qc/9904045

[20] Gomez R, Husa S, Lehner L and Winicour J 2002 Phys. Rev. D 66064019 (Preprint gr-qc/0205038)

[21] Barreto W et al 2005 Phys. Rev. D 71064028 (Preprint gr-qc/0412066)

[22] Winicour J 2005 Living Rev. Rel. 810 (Preprint gr-qc/0508097)

[23] Friedrich H and Stewart J 1983 Proc. R. Soc. A 385 345-71

[24] Bishop N T 1993 Class. Quantum Grav. $10333-41$

[25] Bishop N T, Gómez R, Lehner L and Winicour J 1996 Phys. Rev. D 54 6153-65

[26] Bishop N T, Gómez R, Holvorcem P R, Matzner R A, Papadopoulos P and Winicour J 1996 Phys. Rev. Lett. 76 4303-6

[27] Bishop N T et al 1998 Black Holes, Gravitational Radiation and the Universe ed B R Iyer and B Bhawal (Berlin: Springer) pp 383-408 (Preprint gr-qc/9801070)

[28] Friedrich H 1983 Commun. Math. Phys. 91 445-72

[29] Friedrich H 1986 Commun. Math. Phys. 107 587-609

[30] Friedrich H 1988 Commun. Math. Phys. 119 51-73

[31] Friedrich H 1998 Preprint gr-qc/9804009

[32] Hübner P 1993 Numerische und analytische Untersuchungen von (singulären), asymptotisch flachen Raumzeiten mit konformen Techniken PhD Thesis LMU-München

[33] Hübner P 1996 Phys. Rev. D 53 701-21 (Preprint gr-qc/9409029)

[34] Hübner P 1998 Preprint gr-qc/9804065

[35] Hübner P 1999 Class. Quantum Grav. 16 2823-43 (Preprint gr-qc/9903088)

[36] Hübner P 2001 Class. Quantum Grav. 18 1871-84 (Preprint gr-qc/0010069)

[37] Frauendiener J 1998 Phys. Rev. D 58064002

[38] Frauendiener J 1998 Phys. Rev. D 58064003 (Preprint gr-qc/9712052)

[39] Frauendiener J 2000 Class. Quantum Grav. 17 373-87 (Preprint gr-qc/9808072)

[40] Frauendiener J 2002 Lect. Notes Phys. 604 261-82 (Preprint gr-qc/0207093)

[41] Frauendiener J and Hein M 2002 Phys. Rev. D 66124004 (Preprint gr-qc/0207094)

[42] Husa S 2002 The Conformal Structure of Spacetimes (Lecture Notes in Physics vol 604) ed J Frauendiener and H Friedrich (Berlin: Springer) pp 239-60 (Preprint gr-qc/0204043)

[43] Husa S 2002 Current Trends in Relativistic Astrophysics (Lecture Notes in Physics vol 617) ed L Fernández and L M González (Berlin: Springer) (Preprint gr-qc/0204057)

[44] Frauendiener J 2004 Living Rev. Rel. 7

[45] Pretorius F 2005 Phys. Rev. Lett. 95121101 (Preprint gr-qc/0507014)

[46] Baker J G, Centrella J, Choi D I, Koppitz M and van Meter J 2006 Phys. Rev. Lett. 96111102 (Preprint gr-qc/0511103)

[47] Campanelli M, Lousto C O, Marronetti P and Zlochower Y 2006 Phys. Rev. Lett. 96111101 (Preprint gr-qc/0511048)

[48] Gonzalez J A, Sperhake U, Bruegmann B, Hannam M and Husa S 2007 Phys. Rev. Lett. 98091101 (Preprint gr-qc/0610154)

[49] Diener P et al 2006 Phys. Rev. Lett. 96121101 (Preprint gr-qc/0512108)

[50] Koppitz M et al 2007 Phys. Rev. Lett. 99041102 (Preprint gr-qc/0701163)

[51] Moncrief V 2000 Conformally regular ADM evolution equations Talk at Santa Barbara http://online.itp.ucsb.edu/online/numrel00/moncrief

[52] Andersson L 2002 Lect. Notes Phys. 604 183-94 (Preprint gr-qc/0205083)

[53] Husa S, Schneemann C, Vogel T and Zenginoğlu A 2006 AIP Conf. Proc. 841 306-13 (Preprint gr-qc/0512033)

[54] van Meter J R, Fiske D R and Misner C W 2006 Phys. Rev. D 74064003 (Preprint gr-qc/0603034)

[55] Friedrich H 2002 Lect. Notes Phys. 604 1-50 (Preprint gr-qc/0209018)

[56] Tamburino L and Winicour J 1966 Phys. Rev. 4150 1039-53

[57] Friedrich H 1996 Class. Quantum Grav. 13 1451-69

[58] Friedrich H and Rendall A D 2000 Lect. Notes Phys. 540 127-224 (Preprint gr-qc/0002074)

[59] Choquet-Bruhat Y 1952 Acta Math. 88 141-225

[60] Friedrich H 1985 Commun. Math. Phys. 100 525-43

[61] Andersson L, Chruściel P T and Friedrich H 1992 Commun. Math. Phys. 149 587-612

[62] Chruściel P T and Delay E 2002 Class. Quantum Grav. 19 L71-9 (Preprint gr-qc/0203053)

[63] Corvino J and Schoen R 2003 Preprint gr-qc/0301071

[64] Friedrich H 2004 The Einstein Equations and the Large Scale Behaviour of Gravitational Fields ed P Chruściel and H Friedrich (Basel: Birkhauser) pp 121-203 
[65] Stewart J 1991 Advanced General Relativity (Cambridge: Cambridge University Press)

[66] Newman E and Penrose R 1962 J. Math. Phys. 3566

[67] Penrose R and Rindler W 1984,1986 Spinors and Spacetime (Cambridge: Cambridge University Press)

[68] Lindblom L, Matthews K D, Rinne O and Scheel M A 2008 Phys. Rev. D 77084001 (Preprint arXiv:0711.2084)

[69] Andersson L and Chruściel P 1993 Phys. Rev. Lett. 1970 2829-32

[70] Andersson L, Moncrief V and Friedrich H 1994 Comm. Math. Phys. 161 533-68

[71] Zenginoğlu A 2008 Class. Quantum Grav. 25145002 (Preprint arXiv:0712.4333)

[72] Bishop N T, Gomez R, Lehner L, Maharaj M and Winicour J 1997 Phys. Rev. D $566298-309$ (Preprint gr-qc/9708065)

[73] Bishop N T and Deshingkar S S 2003 Phys. Rev. D 68024031 (Preprint gr-qc/0303021)

[74] Isaacson R A, Welling J S and Winicour J 1983 J. Math. Phys. 24 1824-34

[75] Friedrich H 1998 J. Geom. Phys. pp 83-163

[76] Valiente Kroon J A 2004 Commun. Math. Phys. 244 133-56

[77] Valiente Kroon J A 2004 Commun. Math. Phys. 251 211-34 (Preprint gr-qc/0309016)

[78] Valiente Kroon J A 2006 Class. Quantum Grav. 233593 (Preprint gr-qc/0605056) 